\title{
LA NAISSANCE DE L'ÉLITE FRANCOPHONE TCHÈQUE (EXEMPLE DE TRADUCTEURS TCHĖQUES JAROSLAV VRCHLICKÝ, HANUŠ JELÍNEK ET LEURS CONTEMPORAINS)
}

\author{
Zuzana Raková
}

\begin{abstract}
Frankofile and francophone elite of the Czech society has played since the $19^{\text {th }}$ century an important role in mediating the Czech-French relationships. On the example of two importants translators, Jaroslav Vrchlický and Hanuš Jelínek and their contemporaries, the paper follows the birth of Czech French-speaking cultural and political elite from the 70s of the $19^{\text {th }}$ century to 1918 .

Key words: francophony; francophilie; Czech translators; Czech elite.

Résumé : Les élites tchèques francophones et francophiles ont joué un rôle très important surtout dès la moitié du XIX siècle, comme médiateurs des rapports franco-tchèques. L'article aborde la naissance des élites tchèques francophones dès 1870 jusqu'en 1918 sur l'exemple de traducteurs tchèques Jaroslav Vrchlický, Hanuš Jelínek et de leurs contemporains.

Mots-clés : Francophonie ; francophilie ; traducteurs tchèques ; élite tchèque.
\end{abstract}

\section{Introduction}

Les élites tchèques francophones et francophiles ont joué un rôle très important surtout dès la moitié du XIXe siècle jusqu'en 1918, comme médiateurs des rapports francotchèques. À partir des années 1860, la nation tchèque cherchait une alternative culturelle qui lui permette de s'émanciper de l'influence dominante germanique culturelle et scientifique ; c'est du côté de la France qu'une partie de la nouvelle génération littéraire se retournait. La naissance du système parlementaire en Autriche et la démocratisation de la vie politique tchèque ont encore renforcé ce mouvement émancipateur antigermanique de la société tchèque.

L'orientation francophile se manifesta aussi par une augmentation progressive du nombre des traduction du français. Les Májovci, écrivains réunis autour de l'almanach Máj, dont Josef Václav Frič, Jan Neruda, Vítězslav Hálek et Ferdinand Schulz traduisaient par exemple les chansons de Pierre-Jean Béranger (Pfaff, 1996 : 53-54). 


\section{L'œuvre traductologique de Vrchlický}

Mais le rôle essentiel dans l'introduction de la littérature française dans le milieu tchèque appartient à cette époque-là aux Lumírovci. Leur chef de file était sans aucun doute Jaroslav Vrchlický qui traduisait la poésie française contemporaine et les pièces de théâtre et que nous pouvons considérer comme l'un des plus grands traducteurs tchèques du français. Vrchlický (1853-1912) apprit le français en prenant des cours particuliers au lycée de Klatovy vers la fin des années 1860 (Reznikow, 2002 : 633). Après son arrivée à Prague en 1873, il s'inscrivit à la faculté des Lettres, et il eut bientôt l'occasion de se perfectionner en français en donnant des cours privés de tchèque à Ernest Denis, lors de son premier séjour pragois de 1872 à 1875 (Chrobák, 2003 : 99). En 1875, Vrchlický entra comme précepteur chez le comte Montecuccoli-Laderchi et ainsi, il apprit l'italien, ayant passé une année à Livourne et dans les environs de Modène. De retour à Prague en 1877, il exerçait pendant seize ans la fonction de secrétaire de l'École Polytechnique tchèque et se consacrait à la création artistique qu'il accompagnait de l'activité traductrice. En 1893, il fut nommé professeur de littérature comparée à l'Université de Prague (Jelínek, 1912 : 158). Son tout premier livre publié n'était pas une œuvre originale, mais une anthologie de poèmes de Victor Hugo, Poèmes (Básně, 1874). Victor Hugo lui était particulièrement proche par son vers pathétique, la variété des sujets, la couleur des images poétiques et la foi en l'avenir de l'humanité. Dans sa première période de création littéraire, Hugo était son poète de prédilection qui influença sa propre création poétique : la Légendes des siècles (traduit par Vrchlický comme Legenda véků) l'inspira à composer son propre cycle épique, intitulé Fragments de l'épopée (Zlomky epopeje).

Vrchlický, en tant que représentant le plus important des écrivains autour de la revue Lumír, avait une place prépondérante dans les traductions de la poésie française, tant dans le choix que dans la stratégie traductologique, à partir des années 1870 jusqu'à la fin du XIX ${ }^{e}$ siècle. Le nombre de poésies qu'il avait traduites avant 1893, soit 2356 poèmes de 383 poètes différents (Jelínek, 1912 : 168), montre suffisamment sa grande fécondité traductologique.

L'admiration de Vrchlický pour Hugo ne l'a cependant jamais empêché de traduire d'autres poètes français. Il tenait en grande estime les poètes parnassistes, tel Charles Leconte de Lisle, Théodore de Banville, José-Maria de Hérédia, Sully-Prudhomme et Mme Ackermann, avec lesquels il partageait l'opinion sur la position de l'art et qui appliquaient les mêmes exigeances formelles à la création artistique. Il était un fervent admirateur et interprète dévoué de poètes romantiques Théophile Gautier, Alfred de Musset, Alfred de Vigny. Il faut mentionner avant tout ses deux anthologies de la poésie française, La poésie française des temps modernes (Poesie francouzská nové doby, 1877), et Les poètes français modernes (Moderní básníci francouzští, 1893), contenant près de 700 pièces traduites du français, allant de François Villon jusqu'aux symbolistes.

Vrchlický introduisit dans le contexte littéraire tchèque aussi les poètes maudits, Verlaine, Rimbaud, Mallarmé, Maeterlinck (publiés dans les anthologies), et en collaboration avec Jaroslav Goll, il publia un choix de Fleurs du mal de Charles Baudelaire. Malgré sa prédilection pour la poésie française, Vrchlický traduisit aussi des œuvres prosaïques françaises dont les romans d'Alexandre Dumas père (Les Trois mousquetaires), de Balzac (La femme de trente ans, Třicetiletá, 1886), d'Anatole France et de Maupassant, et des pièces de théâtre dont l'Avare (Molière), le Cid (Corneille), Hernani (V. Hugo), Cyrano de Bergerac (Edmond de Rostand). 


\section{Les traductions prosaïques de la fin du siècle}

Mais malgré l'effort des Májovci et Lumírovci pour faire connaître la littérature française en Bohême et Moravie, ce n'est que dès les années 1880 et 1890 que date l'intérêt plus massif pour la littérature française et surtout sa diffusion parmi les lecteurs grâce aux traductions tchèques, avec l'exception faite pour la poésie qui était traduite systématiquement dès 1875 par Vrchlický. En somme, l'influence de la littérature française sur la création littéraire tchèque demeurait relativement faible encore à la fin des années 1880, comme en témoigne le futur critique littéraire, František Václav Krejčí, contemporain de F. X. Šalda : la génération littéraire des années 1870 et 1880 connaissait Victor Hugo, mais elle ne connaissait pas Balzac ou Stendhal ; elle connaissait Leconte de l'Isle, mais ne connaissait pas Baudelaire ; elle connaissait Daudet, mais ne connaissait pas Flaubert ou les Goncourt. (Krejčí, 1902, Reznikow, 2002 : 637) Ce n'est qu'à partir des années 1890 que les naturalistes, dont Zola, mais aussi Flaubert et Maupassant, sont traduit systématiquement.

L'intérêt croissant pour la littérature française dès le début des années 1890 s'explique par plusieurs phénomènes coïncidant : la France représentait le centre culturel européen et la source de courants artistiques modernes dont le naturalisme et le symbolisme. Mais jusque dans les années 1880, une grande partie des œuvres littéraires françaises n'étaient connues aux Tchèques que grâce aux traductions allemandes, parues dans la Reklamsuniversal-bibliothek (Krejčí, 1989 : 96-100). Cela provoquait une réaction nationale forte vers la fin du siècle, à l'époque des luttes politico-nationales exacerbées. La volonté de s'émanciper de l'influence scientifique et littéraire allemande était à cette époque-là facilitée par la diffusion de l'enseignement public du français. Le système scolaire autrichien introduisit déjà entre 1869 et 1874 dans certains établissements secondaires l'enseignement obligatoire du français. Mais le nombre d'élèves tchèques qui en furent concernés étaient encore très faible dans les années 1870 et 1880 . Ce n'est qu'avec l'élargissement progressif du réseau des écoles secondaires tchèques entre 1870 et 1914, que le système commençait à produire un nombre croissant de Tchèques francophones parmi lesquels les futurs traducteurs de la littérature française se recrutaient-ils (Raková, 2009 : 38-41).

Cela explique pourquoi l'arrivée de la génération de la Moderne tchèque dès 1890 dans la littérature coïncide avec le début de la période des grands projets éditoriaux dans la traduction tchèque du français. L'objectif de cette activité éditoriale était de faire paraître dans les versions les plus complètes possibles surtout les traductions des auteurs réalistes. Les grands romanciers étaient traduits à partir des années 1890, dont Balzac et Flaubert (Jan Třebický, Paní Bovaryová, 1892; František Václav Krejčí, Salambo, 1896; Stanislav Mašek, Sentimentální výchova, 1898), Maupassant (Václav Hladík, Miláček, 1896) et les frères Goncourt (Pavla Moudrá, Germinie Lacerteuxová, 1898). Parmi les auteurs traduits figurait aussi Victor Hugo (Emanuel z Čenkova, Bídníci, 1897-1899). Certains parmi les grands projets éditoriaux initiés avant 1914 n'étaient achevés que pendant la période de la Première république tchécoslovaque. Ainsi, la Bibliothèque de Jan Otto faisait paraître des traductions prosaïques : Maupassant, publié en vingt-quatre volumes entre 1909 et 1913, Zola, publié en trente volumes entre 1908 et 1927, Balzac en dix volumes entre 1910 et 1918, Flaubert en six volumes (1919-1929) et en douze volumes (1930), Anatole France en vingt-sept volumes (1925-1935) et bien d'autres (Hrala, 2002 : 100-101).

Le critique littéraire František Václav Krejčí (1867-1941) se souvenait ainsi, dans ses mémoires, de son apprentissage du français dans une ville de la Bohême orientale, et 
de ce que la littérature française représentait pour lui et sa génération dans les années 1880-1890 :

J'apprends le français. Je me suis procuré la grammaire et le livre d'exercice de Herzer, et chaque jour, j'étudie au moins une heure. Le travail est facile, avance rapidement et me remplit d'espoir que ses résultats vont $m$ 'introduire bientôt dans le monde de la culture française. ... Je n'ai évidemment pas appris le français pour pouvoir parader en le parlant, mais pour pouvoir lire et comprendre des œuvres littéraires françaises. ... Pour la vie d'alors, le français n'avait pas encore d'importance pratique chez nous, s'y consacraient seulement les professeurs et les instituteurs qui l'enseignaient comme matière à l'école. À part les classes d'école, on ne pouvait entendre le français que dans les centres de la soi-disante meilleure société, où les dames issues de l'école supérieure de jeunes filles ou ayant appris la conversation française auprès de véritables Françaises, attiraient l'attention sur elles en parlant français. Chez nous, les Français des deux sexes, comme dans les autres pays à l'Est du Rhin, appartenaient à la société élégante voire aristocratique, comme c'est par exemple Monsieur Triquet dans Eugène Oniéghine. Le français était, pour le dire brièvement, une langue de demoiselles, une sorte de luxe social, la marque du goût le plus sublime. ... Apprendre le français dans des buts littéraires est devenu entretemps un besoin. ... Surtout avec l'entrée de Vrchlický en littérature, tout a changé. Il a supprimé la dépendance de la poésie tchèque de l'influence allemande. ... On a ressenti la nécéssité de nouer des rapports directs avec l'Occident. Vrchlický charmait aussi par le fait qu'il était en France et en Italie comme chez soi. ... Et plus encore Julius Zeyer, qui connaissait non seulement les villes françaises avec leurs cathédrales, mais aussi la littérature française contemporaine de même que les légendes populaires moyennageuses. Apprendre le français signifiait alors suivre ces deux poètes dans l'effort d'enrichir sa vie du charme de la langue, qui était la clé de la culture la plus brillante de l'Europe d'alors. ... Avant 1890, chacun qui a voulu chez nous acquérir une largeur d'esprit et s'orienter dans la littérature européenne, devait considérer la connaissance du français comme l'une des clés les plus imporantes. Je suppose que la plupart de mes futurs collègues littéraires étaient assis dans leur jeunesse devant les grammaires françaises autant que moi. ... Chose curieuse, j'apprends une langue dont je n'entends pas une seule parole durant toute l'année, une langue dont je ne connais absolument pas la mélodie, le son, lorsqu' on la parle. (Krejčí, 1989: 96-100)

Le témoignage de F. V. Krejčí est assez symptomatique pour l'époque et illustre bien les difficultés auxquelles se heurtaient les intellectuels tchèques désirant apprendre le français, surtout dans les villes petites ou moyennes. Les écoles secondaires tchèques offrant le français dans leurs programmes n'étaient pas dans toutes les villes de la Bohême et de la Moravie. Beaucoup d'écrivains, artistes ou même traducteurs apprirent le français soit comme autodidactes, soit en prenant des cours particuliers auprès d'un Français de la ville.

Aussi Hanuš Jelínek, l'un des plus importants médiateurs franco-tchèques, évoquet-il dans ses mémoires Jaroslav Vrchlický qui, tout en étant excellent traducteur de la poésie française en tchèque, avait du mal à s'exprimer en français, de même qu'un autre poète tchèque, Viktor Dyk, qui était capable de lire en français mais « la connaissance pratique de la langue française lui manquait et la conversation en français lui posait de graves difficultés » (Jelínek, $1947: 489)$.

\section{Un tournant décisif pour la francophonie tchèque}

Quant à la francophonie de l'élite politique et culturelle tchèque, elle fut assez rare durant le XIXe siècle. Seulement quelques hommes politiques dont František Palacký, František Ladislav Rieger, Josef Václav Frič possédaient une connaissance du français, 
La naissance de l'élite francophone tchèque...

et encore chez Palacký il s'agissait d'une connaissance plutôt passive. Sinon, la connaissance pratique du français était le domaine des artistes tchèques vivant ou ayant vécu en France, tels Soběslav Pinkas, Jaroslav Čermák, Václav Brožík, Zdeňka Braunerová, Luděk Marold, Julius Zeyer, Antonín Chitussi, František Bílek ou Alphonse Mucha, et des professeurs de français, bien évidemment.

Ce n'est qu'au tournant des $\mathrm{XIX}^{\mathrm{e}}$ et $\mathrm{XX} \mathrm{X}^{\mathrm{e}}$ siècles qu' une connaissance solide du français écrit et parlé se diffuse parmi les jeunes représentants de l'intelligentsia tchèque. À la romanistique tchèque de Prague étudiait, dans le séminaire de langues romanes de Jan Urban Jarník vers 1900, la génération de Hanuš Jelínek ; parmi ses collègues figuraient Maxmilián Křepinský, Prokop Miroslav Haškovec, Ferdinand Špíšek, Karel Titz, František Žákavec, Rudolf Kepl, Otakar Šimek et d'autres. Il s'agissait des jeunes gens nés entre 1875 et 1879 . Plusieurs de ces jeunes hommes devinrent par la suite professeurs de français dans le secondaire, professeurs universitaires en langues romanes ou d'autres disciplines, certains se consacrèrent au développement des rapports franco-tchèques.

\section{Le personnage de Jelínek}

L'un des hommes les plus actifs dans ce domaine était Hanuš Jelínek (1878-1944), le principal médiateur des rapports franco-tchèques dès la Belle Époque. Né à Příbram où il a commencé en 1894 à apprendre le français comme autodidacte, d'une grammaire française rédigée en allemand. Il s'inscrivit à l'Université tchèque à Prague initialement en philologie allemande et slave, optant par la suite pour la philologie romane, sous l'influence du lecteur de français Georges Mohl et du poète Jaroslav Vrchlický qui donnait à l'Université des cours sur la littérature comparée. Jelínek effectua deux séjours d'études d'un an et de six mois à Paris en 1897 et en 1898, où il entra en contact avec Ernest Denis, Louis Léger et aussi la colonie tchèque. Les séjours prolongés et répétés en France ont porté leur fruit : la maîtrise parfaite du français courant ainsi que l'élégance de la tenue devinrent attributs indissociables de Hanuš Jelínek, d'après le témoignage de son collègue de classe de l'Université pragoise, Ferdinand Špíšek. Jelínek qui avait fréquenté des cours à la Sorbonne et des boîtes de Montmartre, se désintéressait « des cours de Jarník consacrés à la grammaire historique des langues romanes, qui ne pouvaient pas convenir au jeune 'étudiant parisien'. » Il pouvait se le permettre parce que son niveau de français était l'objet d'envie de plusieurs de ses collègues tchèques (Jelínek, 1947 : 196-197).

Après avoir fini ses études, Jelínek est devenu professeur du secondaire, habilité en français et en allemand. Il enseignait le français à l'Académie de commerce tchéco-slave à Prague dès le début du XXe siècle jusqu'en 1918. En plus, il a obtenu le doctorat de littérature. En 1909-1910, il donna des cours sur la littérature tchèque à la Sorbonne, à l'initiative d'Ernest Denis qui avait eu l'idée de faire professer ce cours pour compléter la formation de ses étudiants : Denis enseignait aussi l'histoire des Pays tchèques. Jelínek fut libéré de son école et il avait un congé payé pendant toute l'année scolaire. Pour le remplacer, le proviseur de l'Académie de commerce Řeřábek appella au poste de professeur de français le jeune docteur ès-lettres et ès-droit Edvard Beneš. Le cours de Jelínek se réalisa au premier semestre de 1910 et fut un succès par le nombre d'étudiants (jusqu'à 70) et par l'écho dans la presse française. Ce succès se traduisit par la publication de la Littérature tchèque contemporaine de Jelínek au Mercure de France en 1912, avec une préface d'Ernest Denis. On y trouve des traductions de la poésie de K. H. Mácha, J. Neruda, J. V. Sládek, P. Bezruč, J. S. Machar, O. Březina, A. Sova etc. Jelínek déploya un 
grand effort pour faire connaître la littérature tchèque en France. Il écrivit de nombreux articles sur la littérature tchèque dans différentes revues. Son œuvre la plus importante représentent ses trois volumes consacrés à l'Histoire de la littérature tchèque: I. Des origines à 1950 (Paris, 1930), II. De 1850 à 1890 (Paris, 1930), III. De 1980 à nos jours (Paris, 1935). Jelínek traduisit en français des pièces dramatiques, dont Un père d'Alois Jirásek qui fut montée en 1923 à Bruxelles comme la première pièce tchèque sur une scène francophone (Reznikow, 2002 : 501-512) et était ensuite publiée dans la Gazette de Prague en 1924. La traduction de R.U.R. de Karel Čapek, était publié à Paris en 1924. En 1930, on édita à Paris son recueil Anthologie de la poésie tchèque.

Jelínek faisait connaître également la littérature française au public tchèque, comme traducteur infatigable. Il traduisait en tchèque deux séries de chansons et ballades françaises (Zpěry sladké Francie, 1925 et 1930). En 1925, il édita un recueil consacré à la poésie française contemporaine, qui influença la traduction poétique tchèque en général $(D e$ la poésie française contemporaine, Ze současné poezie francouzské, Ilek, 1982 : 33). Il traduisit également des œuvres en prose et des pièces de théâtre (Molière, Stendhal, Musset, Roger Martin du Gard, Henri Barbusse : Le Feu, 1917). Ce dernier roman critiquant la guerre, provoqua des polémiques politiques et littéraires en Tchécoslovaquie; l'œuvre représentait aussi un défi pour le traducteur ; il s'agissait de trouver des équivalents de la langue parlée, du français familier avec des expressions argotiques (Stavinohová, 1995 : 107).

Que la France incarnait un modèle culturel, scientifique et politique alternatif envers l'influence prédominante allemande, en témoignent ces paroles d'un farouche francophile, contemporain de Jelínek, Emanuel z Čenkova, adressées à la jeunesse tchèque en 1909, dans la Revue des étudiants progressistes:

La civilisation française sera toujours chez nous un correctif affinant et ennoblissant de l'influence allemande. ...Vous savez comment les Allemands aspirent à la ruine de notre langue tchèque. Cultivez donc assidûment, quotidiennement et systématiquement la langue française. Quinze minutes de travail sérieux tous les jours portera ses fruits dans quelques années. Vous, futurs hommes de la politique, de l'art, de la science et du commerce tchèques, ne diffusez pas dans les rapports internationaux, la langue de nos ennemis, employez le français. Ainsi, vous ferez reculer l'influence allemande dans le monde et vous renforcerez l'influence de nos amis. (Čenkov, 1909: 3-4)

\section{Conclusion}

Malgré cette propagation de l'emploi du français dans la communication internationale par des francophiles tchèques, la connaissance active de la langue française resta pendant longtemps très élitiste : bien apprendre le français parlé était possible soit d'une gouvernante ou d'un professeur français ou francophone, ou bien en passant un certain temps en France ou dans un pays francophone. Les deux possibilités n'étant pas accessibles à tout le monde, la connaissance de la culture française était facilitée par le travail des traducteurs tchèques qui ont servi d'intermédiaires entre les deux cultures.

Les relations entre les deux pays avaient, en plus de leur importance culturelle, une dimension politique surtout au XIX $X^{\mathrm{e}}$ siècle et jusqu'en 1918, parce que la France jouait un rôle non négligeable dans l'émancipation culturelle et politique de la nation tchèque du joug allemand. La plupart de la société tchèque étant francophile, mais pas forcément francophone, l'essor de l'influence culturelle française résultait surtout du travail assidu 
La naissance de l'élite francophone tchèque...

de plusieurs traducteurs, dont Jaroslav Vrchlický et Hanuš Jelínek. Le travail de leurs contemporains francophones, le plus souvent professeurs de l'enseignement secondaire ou universitaire, journalistes ou artistes, était également décisif pour le rapprochement mutuel des deux nations au tournant des XIX et $X X^{\mathrm{e}}$ siècles. À l'effort de plusieurs francophiles tchèques, tel Soběslav Pinkas, Václav Hladík, Henri Hantich, Emanuel z Čenkova, Hanuš Jelínek ou Ferdinand Špíšek, s'ajoutait l'intérêt croissant pour la nation tchèque de la part de quelques slavisants, professeurs universitaires et journalistes français, dont surtout Louis Léger, Ernest Denis, Albert Sorel, André Chéradame, Louis Eisenmann ou Jules Legras.

\section{Bibliographie :}

ARChIVEs de l'Académie des sciences de la République tchèque : fonds personnels de Ferdinand Špíšek 1950-1965 : Mémoires, souvenirs, n 1, 2 et 3, Prague.

Catalogue des ouvrages français traduits en tchèque, publiépar l'Alliance française de Prague à l'occasion de l'Exposition Universelle de Paris en 1889 et comprenant les traductions déjà parues ou terminées en manuscrit seulement, à la fin de l'an 1888. (1889), Prague: Alliance française de Prague. - Přehled překladů z jazyka francouzského do češtiny. Vydán za príčinou světové výstavy pařížské roku 1889 péčí Alliance française v Praze. Obsahuje veškeré překlady uveřejněné aneb v rukopise ukončené do konce roku 1888. (1889) Praha : Alliance française v Praze.

ČENKOVA, Emanuel z (1909), « List mládeži (O stycích česko-francouzských) », Časopis pokrokového studentstva 13.1, 3-4.

ČernÝ, Václav (1994), Paměti I 1921-1938, Brno : Atlantis.

Hrala, Milan, éd. (2002), Kapitoly z dějin českého překladu, Praha : Karolinum.

CHRoвÁK, Tomáš (2003), « Ernest Denis - sa vie et son temps », in : CHROBÁK, Tomáš, OlšÁKová, Doubravka (éds.), Ernest Denis, Praha : Eva, 99-142.

ILEK, Bohuslav (1982), "Le passé et le présent de la traduction tchèque », in : La Traduction en Tchécoslovaquie, Prague, Fonds littéraire tchèque et Fonds littéraire slovaque, $5-48$.

JeLíneK, Hanuš (1947), Zahučaly lesy, Praha : Fr. Borový.

JELínEK, Hanuš (1912), La littérature tchèque contemporaine, Paris : Mercure de France, 1912.

KREJČí, František Václav (1989), Konec století. Výbor z pamětí, Praha : Československý spisovatel.

KREJČí, František Václav (1902), « Les influences françaises sur la nouvelle littérature tchèque », in : Hipman, Charles (éd.), Les Tchèques au XIXe siècle, La Nation tchèque, Prague, non paginé.

NoËL, Léon (1982), La Tchécoslovaquie d'avant Munich, Paris : Institut d'études slaves et Publications de la Sorbonne.

Pfaff, Ivan (1996), Česká přináležitost k Západu v letech 1815-1878. K historii českého evropanství mezi vídeňským a berlínským kongresem, Brno.

Pistorius, Georges (1957), Destin de la culture francaise dans une démocratie populaire : la présence francaise en Tchécoslovaquie (1948-1956), Paris : Les Îles d'Or.

RAKOvÁ, Zuzana (2009), Francophonie de la population tchèque 1848-2008. Olomouc: FF UP, thèse non publiée.

REZNIKOW, Stéphane (2002), Francophilie et identité tchèque (1848-1914), Paris : Honoré Champion. 
STAVINOHOVÁ, Zdeňka (1995), « Deux traducteurs tchèques importants et leur grand rôle culturel », in : AUC, Philologica 2, Translatologica Pragensia VI, Praha: FF UK, 105-108.

Zuzana Raková

Ústav románských jazyků a literatur

Filozofická fakulta

Masarykova Univerzita v Brně

Gorkého 7

60200 Brno

République tchèque

RakovaZ@seznam.cz 